\title{
Simulations of 3D detectors
}

\section{Gabriele Giacomini ${ }^{1}$, Claudio Piemonte}

FBK-Silicon Radiation Sensors

via Sommarive 18, I-38123 Trento, Italy

E-mail: ggiacomini@fbk.eu, piemonte@fbk.eu

\section{Gian-Franco Dalla Betta, Marco Povoli}

Dipartimento di Ingegneria e Scienza dell'Informazione

Università degli Studi di Trento

and

INFN Sezione di Padova, Gruppo Collegato di Trento,

via Sommarive 14, I-38123 Trento, Italy

E-mail: dallabe@disi.unitn.it, povoliedisi.unitn.it

3D detectors, in which the $n$ and $p$ electrodes are columns etched through the silicon substrates, have proven high radiation tolerance and thus are one of the most suitable candidates for harsh radiation environments, such as vertex detectors. Being the process much more complicated than the planar one, over the years, several simplified 3D families have been studied and fabricated. In this context, TCAD simulations are an excellent tool to predict the behaviour and performance of new detector concepts. In this paper we give an overview of this simulation activity mainly focussing on the 3D FBK technology. We show that it is possible to reproduce also unexpected phenomena, such as the observed charge multiplication in highly irradiated devices.

The 20th Anniversary International Workshop on Vertex Detectors - Vertex 2011

Rust, Lake Neusiedl, Austria (June 19-24, 2011)

$1 \quad$ Speaker 


\section{Introduction}

3D detectors were proposed in 1997 by Sherwood Parker and collaborators [1] [2] as silicon sensors in which the electrodes are columns etched through the silicon substrate. In a planar detector, the $p$ and $n$ electrodes are usually placed on the opposite sides of the silicon wafer, at a distance which cannot be easily reduced below $200 \mu \mathrm{m}$, due to issues related to the handling of thin wafers is a clean room ${ }^{1}$. In 3D detectors the electrode spacing is decoupled from the wafer thickness, and is a matter of layout. The advantages of this approach are: low collecting distances, low operational voltages and fast collection times. These features are mandatory in harsh radiation environments, such as the vertex detectors of modern high luminosity colliders. Here, the radiation damage increases the effective $p$-type doping concentration of the silicon bulk: the depletion voltage of a $200 \mu \mathrm{m}$ thick heavily irradiated planar sensor can easily exceed $1000 \mathrm{~V}$ while the small electrode spacing in a 3D detector allows full depletion already at 100-200 V. Moreover, radiation damage creates traps into the bulk, making fast collection times and short collection distances mandatory and, at the same time, rendering large depletion depths useless. Superior radiation hard performances have already been obtained, showing a collection of $66 \%$ of the original signal after exposing a 3D sensor at a fluence of $8.8 \mathrm{e} 151-\mathrm{MeV}-\mathrm{n}_{\mathrm{eq}} / \mathrm{cm}^{2}$ (at $100 \mathrm{~V}$ of applied bias) [4]. Irrespective of radiation damage, fast collection time is needed also in applications needing an excellent timing resolution, such as in far forward detectors, where the relative timing of crossing particles can locate their vertex position [5].

This performance enhancement of the 3D has some price: an increase of the capacitance (by a factor 2-3, depending on the layout) with respect to the planar and, moreover, a more complex fabrication. In recent years, CNM (Centro National de Microelectronica, Barcelona, Spain) and FBK (Fondazione Bruno Kessler, Trento, Italy) have initiated R\&D in the fabrication of large area 3D detectors, as the sensitive part of a system read-out by the FE-I4 chip [6] [7], for the ATLAS IBL (Insertable B-Layer) [8]. This barrel, being very close to the beam pipe, will undergo a heavy radiation exposure during the foreseen 10-year operation (the estimated fluence will be up to $5 \mathrm{e} 151-\mathrm{MeV}-\mathrm{n}_{\mathrm{eq}} / \mathrm{cm}^{2}$ ). 3D productions so far have been intended only for prototyping, while a final decision whether the IBL will be entirely fitted with standard planar n-on-n sensors (produced by CiS, Germany) or also partially with 3D from both CNM and FBK will be made in February 2012.

Only a few foundries all around the world have processed such types of devices. Beyond the above mentioned CNM and FBK, there are the Stanford Nanofabrication Facility (Stanford, USA), Sintef (Oslo, Norway) and VTT (Helsinky, Finland).

To simplify the fabrication process with respect to the original one proposed by Kenney [9], modified 3D structures have been proposed. For example, CNM chose, for IBL prototypes, a 3D option with not full passing columns (i.e. the columns are not etched throughout the whole

A way for processing thin active substrates is using epitaxial wafers: the epitaxial layer itself, whose thickness could be as low as 50 micron, is the sensitive volume while the substrate acts as a mechanical support. In the case of a pixel sensor, after the bump-bonding of the sensor to the front-end chip, the substrate is thinned by means of a lapping procedure and then metalized [3]. 
wafer thickness) while FBK 3D sensors evolved from the (not-full-passing) Single-Type Column (STC, Fig. 1(a)) to the (not-full-passing) Double-sided Double-Type columns (DDTC, Fig. 1(b)) and arriving nowadays to the (full-passing, i.e. the columns are etched throughout the whole wafer thickness) Double-sided Double-Type columns (modified DDTC, Fig.1(c)) for the IBL prototypes.

There is also a complexity in the physical behavior of such devices: planar devices have already been extensively studied and are well understood, but this is not the case of 3D sensors whose electrical and dynamic characteristics must be accurately simulated, being far from intuitive. Moreover, a given 3D technology is different from the other and dedicated simulations have to be done. These simulations, as explained later, must be 3-Dimensional (3D).

In this paper, a series of different 3D simulations and their successes in describing various aspects of 3D-sensor behavior will be described.

This paper is organized as follows: in section 2, the TCAD simulator is briefly reviewed. In section 3, a history of the simulations on the subject is reported as well as the fundamental results, with particular attention to the work done on FBK detectors. In section 4, a chosen example of a recent simulation will be extensively presented. Conclusions are drawn in section 5.

\section{The TCAD Simulator}

Before device production, it is much more economical than the fabrication itself, in terms of both money and time, to try to predict its properties by means of numerical simulations. Once the measurement campaign of the final devices has been performed, some unexpected electrical characteristic may come out, both in the static (current/voltage or capacitance measurements) and in dynamic (signal and noise measurements) characterization and then need an adequate interpretation: the simulations can help also in this phase. Thus, simulations provide meaningful feedback, which can be used to start a new technology.

There exist several commercial tools for the numerical simulation of electronic devices. The most popular are essentially two: the SILVACO environment and Sentaurus (by Synopsys). They essentially do the same job, i.e. they solve drift, diffusion, Poisson and other semiconductor equations on a user defined lattice, which is a grid approximating the real device. The higher the number of nodes making up the lattice, the more accurate the simulation results. Even if a real device is, by definition, 3-dimensional, most simulations can be conducted in 2D approximation. In these cases, all vector quantities in the missing coordinate are neglected (set to zero) while scalar quantities are understood to be constant. Consider a case in which the structure dimension in one direction is by far larger than the ones in the other two: for example a strip detector in which the length of the electrode is much larger than both the substrate thickness and the strip pitch. If we are interested in the behavior of the electrical quantities in the middle of the strip, that direction does not need to be simulated, because any quantity in that direction can be treated as constant (or even zero). On the other hand, if we are interested in the tip of the strip, a 3D simulation is needed. 
A 1D simulation is reliable only in the few cases in which two out of three directions can be neglected, i.e. only for two-terminal devices, which are the MOS capacitor and the ideal diode. In these cases, a thorough insight of the physics is provided by the simulator, but this has practically only a didactical application.

In case of 3D detectors, a comprehensive simulation needs normally to be 3Dimensional, since properties varies with depth and since cylindrical symmetry is never obtained in a real 3D device where arrays of columns have to cover a large area.

Indeed, 2D simulations, when used for 3D detectors, provide only approximate results.

Only few quantities can be predicted rather well: one of these is, for instance, the charge collection from a m.i.p. in a 3D detectors with full-passing-columns, once a plane perpendicular to the columns and passing at mid-substrate is simulated. In fact, charge collection properties are little affected by the surfaces in this case. But when aiming at an accurate simulation also accounting for surface effects, that are known to significantly affect some detector properties (e.g., capacitance and breakdown voltage), 3D simulations are required. This was also the case of our 3D-DDTC with non-full-passing columns and of 3D-STC, for which, whatever is the chosen 2D plane, 2D simulations would give only a limited insight, due to the intrinsically 3dimensional structure of the device.

One drawback of 3D simulations is that they usually require high number of nodes, causing very long CPU times even in latest-generation PCs. This problem can be alleviated by exploiting the fact that, often, the elementary cell of a 3D detector can be quite small. In any case, the simulator applies the Neumann boundary conditions, for which the electric field component normal to the interfaces (if charges are absent) is zero: this guarantees that the currents cannot flow through interfaces but only through the contacts. As a consequence of such boundary conditions, a device is mirrored at its interfaces, allowing the full simulation of a large device by only considering its elementary cell.

\section{Overview of 3D simulations}

The publication first introducing 3D devices [1] used 2D numerical simulations to demonstrate the superior properties of 3D detectors, i.e. low operational voltages and short collection times. The simulated structure was $2 \mathrm{D}$ and lying on a plane perpendicular to the columns. The approximation holds only for planes crossing far from the surfaces. The $2 \mathrm{D}$ calculation was sufficient to show and prove the main features of the 3D concept. For more precise calculations, for example of the electric fields, 3D structures must be considered.

In the following, we present $3 \mathrm{D}$ simulations performed by FBK and other groups working on 3D sensor development.

The first generation of 3D sensors fabricated at FBK featured only junction $(n)$ columns (Single Type Column, STC), furthermore such columns were not full passing (Fig. 1 (a)). Substrate $(p)$ contact was supplied at the unpatterned backside. This resulted in an important reduction of the number of technological steps. Simulations, mainly focused on signal formation [10] [11], show a fast collection time for electrons generated in proximity of a column. On the contrary, a very low electric field half-way the $n$-columns cause a rather slow collection of the electrons generated in that position. Indeed, electrons move at first only by diffusion, and only 
in a second time, when entering a high field region, drift rapidly to the columns. Independently of the hit position, signals have quite long tails, due to slow collection of holes, which first move horizontally, following the field lines perpendicular to the columns, up to the mid-points in between the columns and then slowly drift vertically to the backside. Moreover, the applied voltage in excess to full lateral depletion voltage does not help in increasing the electric field in between the grounded columns: it increases only the electric field from the column tip to the backside.

Double-sided Double-Type Column (DDTC) 3D sensors [12][13], aimed at performance enhancement while maintaining a reasonably simple process, were the next generation of 3D at FBK (Fig. 1 (b)). The main issue was to improve the charge collection efficiency, as compared to the one of the old STC batches, in which it was found to be quite poor. In 3D-DDTC, the columns, which are etched from the two sides, stop at a short distance from the opposite side and, thus, have only a partial overlap. Good charge collection properties are found once the distance between the column tip and the opposite surface is small, with only little worsening with respect to 3D sensors having full-passing columns.

In the case of a limited columnar overlap [14][15], only carriers generated in the overlap region are rapidly collected. Indeed, as can be seen in Fig. 2, signal electrons generated here drift to the $n$-column in less than 2 ns. On the other hand, a much slower charge collection is predicted in the regions outside of this overlap, since electric fields are much weaker. An experimental confirmation of the simulations was done by shining laser pulses of different wavelengths onto the detector, allowing to control the depth of generation of the carriers [15].

Simulations played an important role also for the development of 3D-DDTC detectors at CNM. In [16], D. Pennicard et al. discussed the properties of unirradiated 3D-DDTC structures by simulating the electrostatic behavior, the I-V and $\mathrm{C}-\mathrm{V}$ characteristics, the breakdown behavior and the charge collection performances.
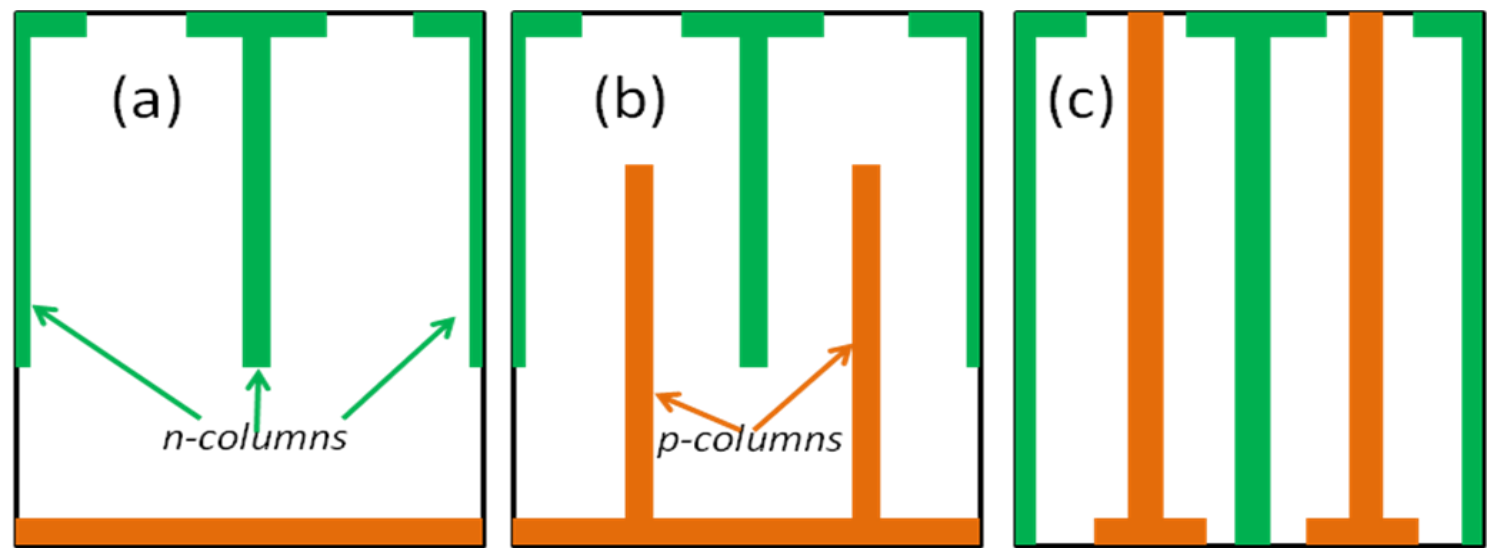

Fig. 1: Sketch of the three families of 3D sensors fabricated at FBK: (a) Single Type Column (STC), (b) Double-sided Double-Type Column (DDTC), (c) Modified Double-sided DoubleType Column (Modified DDTC).

Moreover, some simulations were done increasing the depth of both columns, up to the limit case in which the column pass all the way through the substrate (as in the Modified 3D- 
DDTC detector structure, see Fig. 1(c)). The conclusion was the same as in [12]: the performance is reasonably good once the columns are deep enough.

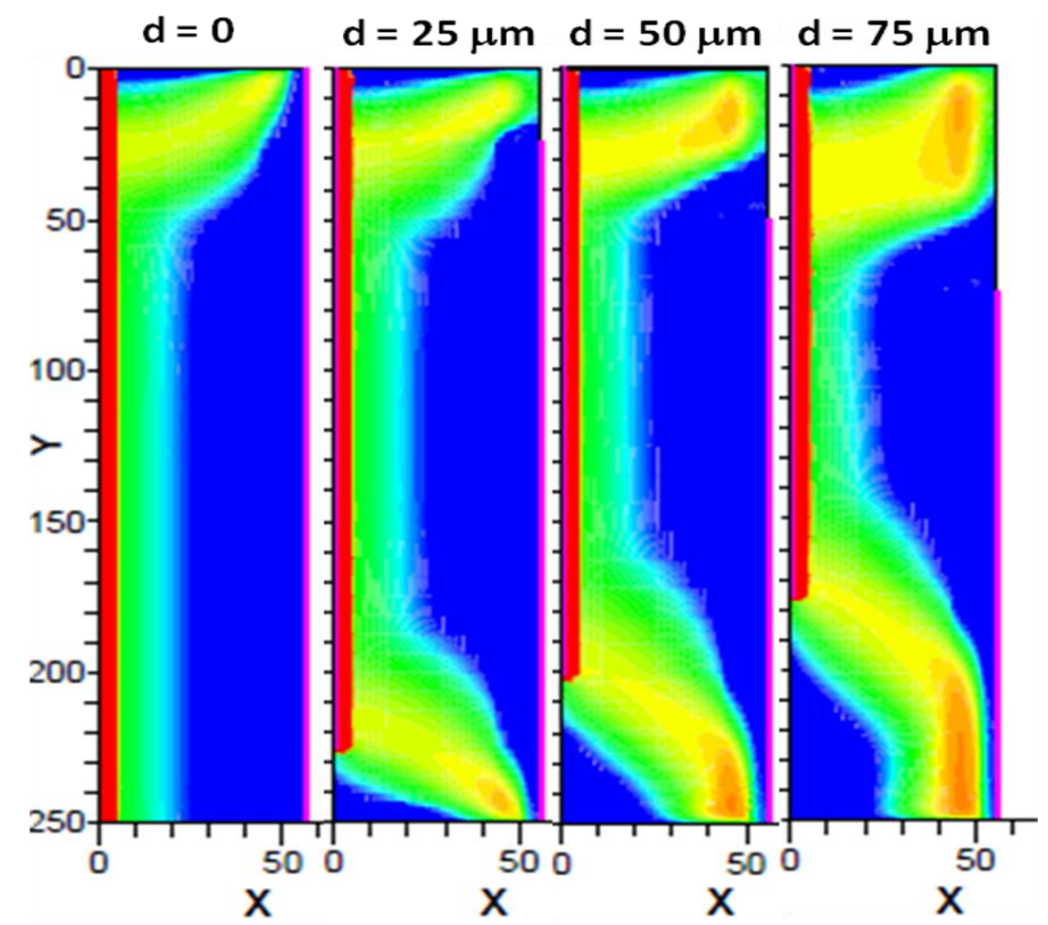

Fig.2: Electrons density concentration, in four different $3 D$ detectors, $2 n s$ after a vertical crossing of a m.i.p. Junction (n) column is in red and at the left side of the slice, ohmic $(p)$ column is in violet at the right side. Four cases are here represented: one 3D Modified DDTC detector (i.e. with full-passing-columns) and three 3D-DDTC detectors with a spacing of 25, 50 and $75 \mu \mathrm{m}$ from the column tips to the opposite surface. Electrons generated in the column overlap region are rapidly collected while the collection of electrons generated outside this region is delayed.

Radiation damage was only taken into account by considering the breakdown voltage in the presence of an increased oxide charge density: 3D-DDTC sensors are more robust with respect to 3D with full passing columns (such as the ones described in [1] or the FBK Modified DDTC) since the p-column tip is not embedded in the interfacial electron layer. These considerations made CNM confident that this 3D approach, with $p$-stop isolation and not full passing columns, can meet IBL requirements.

In another work [17], D. Pennicard et al. considered a Modified 3D-DDTC sensor, irradiated up to $1 \mathrm{e} 161-\mathrm{MeV}-\mathrm{n}_{\mathrm{eq}} / \mathrm{cm}^{2}$. The irradiated device is modeled by inserting traps into the bulk, according to the "Perugia" model [18]. After verifying that the trap model accurately predict the leakage current and the charge collection efficiency of an irradiated planar detector, a variety of different 3D pixel layouts (to be coupled to the FE-I3 read-out chip of the ATLAS experiment [19]), differing for the number of $n$ columns per pixel (ranging from 2 to 8), have been taken into account. CCE, noise, capacitance were simulated to extract the best number of columns per cell. i.e. those maximizing the $\mathrm{S} / \mathrm{N}$ ratio. 

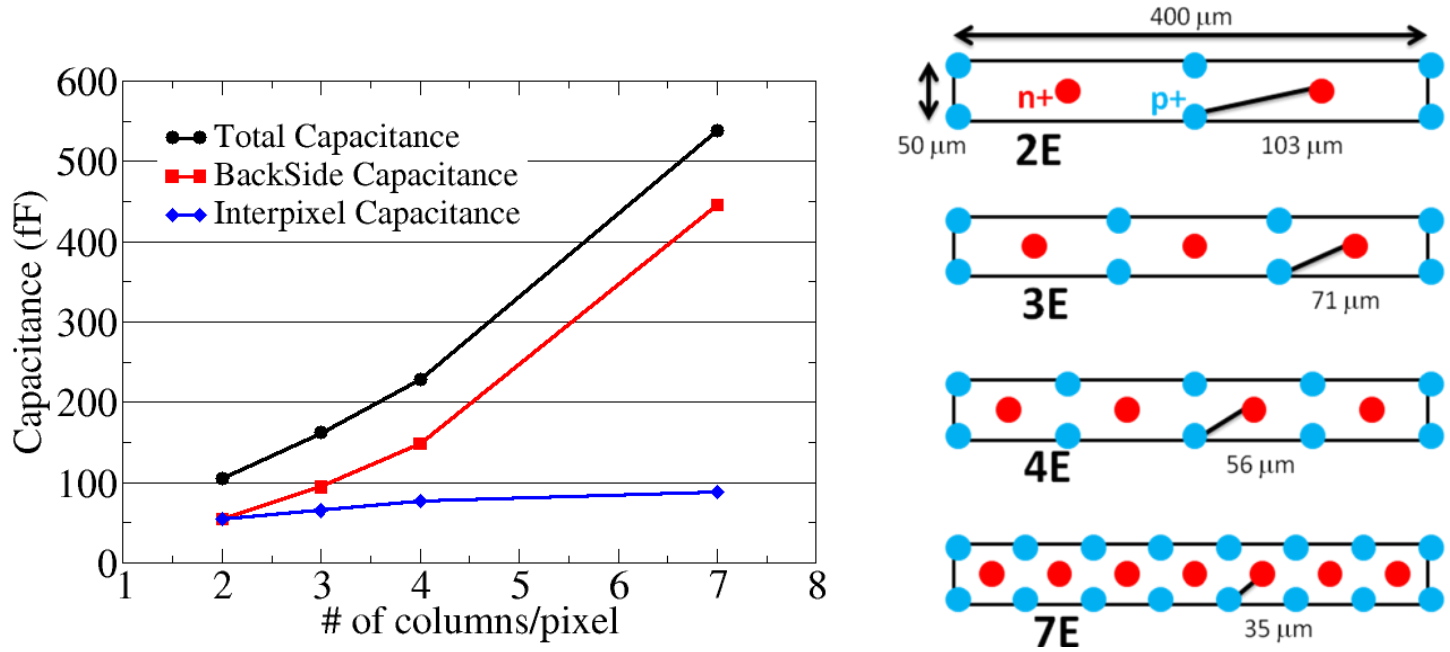

Fig.3 Simulated contributions to the total pixel capacitance of the backside and the interpixel capacitances for 4 types of proposed ATLAS FE-I3 pixels: 2E, 3E, $4 E$ and 7E, having 2, 3, 4 and 7 junction (n) columns per pixel respectively.

With a similar approach, we simulated the pixel capacitance of FBK 3D-DDTC sensors as a function of the number of junction ( $n$ ) columns per pixel [14], and the results were found to be in very good agreement with those of [17]. As can be seen in Fig.3, interpixel capacitance (i.e. the capacitance between the junction $(n)$ columns of the adjacent pixels) is negligible, because of the shielding action of the ohmic-p-columns. On the other hand, the backside capacitance (i.e. the capacitance between the $n$-columns of a single pixel and all the $p$-columns of the sensor) is dominant and significantly increases as the number of columns is increased, resulting in higher noise. On the other hand, a larger number of columns corresponds to lower distance between the columns, so that it is easier to counteract trapping effects and to improve the charge collection efficiency. In this respect, 3,4 $n$-columns per pixel are a good capacitance/charge-collection-efficiency trade-off (indeed 3D-DDTC sensors with these pixel type have been widely used in test beams [20]).

Recently, FBK started an R\&D aimed at the fabrication of 3D sensors to be bump bonded to the FE-I4 chip for the ATLAS IBL. The technology chosen features full passing, Double-sided Double-Type Columns (Modified DDTC, see Fig.1(c)) [21]. IBL dictates an inactive area not exceeding $225 \mu \mathrm{m}$ at the sensor edge. This spec can be met by implementing an active edge, as already proposed by Parker [22][23]. The main drawback is that it requires a support wafer (making it incompatible with a double-side process), which is not easy to remove, expensive and thus not advisable for a mass production. As an alternative, a border region featuring multiple rows of ohmic $(p)$ columns was developed. Simulations, later confirmed by measurements [24], showed how the depletion region, extending from the outermost junction $-n$ - columns, stops at the first $p$-column row, allowing a very short safe distance (order of 100 $\mu \mathrm{m})$ between active area and scribe line. The possibility to implement slim edges comes out to be another strong motivation to prefer 3D to planar sensors. 


\section{A selected Example: Multiplication effects on irradiated devices}

In this paragraph we show one of the latest simulations we performed on the 3D-DDTC technology. The sensor under investigation was a 3D strip detector (i.e. rows of junction columns are shorted together) fabricated at FBK and featuring: a 300- $\mu$ m thick FZ $n$-substrate, $p$-junction columns $190 \mu \mathrm{m}$ deep, and $n$-ohmic column 160 um deep (the overlap was then only $50 \mu \mathrm{m})$. The $80 \mu \mathrm{m} \times 80 \mu \mathrm{m}$ elementary cell has a $p$-column at the center and $n$-columns at the corners. The detector was irradiated with $24 \mathrm{MeV}$ protons at Karlsruhe synchrotron, up to a fluence of $1 \mathrm{e} 151-\mathrm{MeV}-\mathrm{n}_{\mathrm{eq}} / \mathrm{cm}^{2}$. The measured collected charge released by a m.i.p (Fig. 4) gradually increases with bias in the low voltage range as the substrate gets more and more depleted and as the radiation induced trapping becomes less important because of the shorter collection times [13]. Then, it starts increasing exponentially at a voltage of about $200 \mathrm{~V}$, and finally exceeds the collected charge of the un-irradiated sensor. The same trend is closely followed by the leakage current (not shown in Fig. 4). It looks like if some charge multiplication effect is present into the bulk.

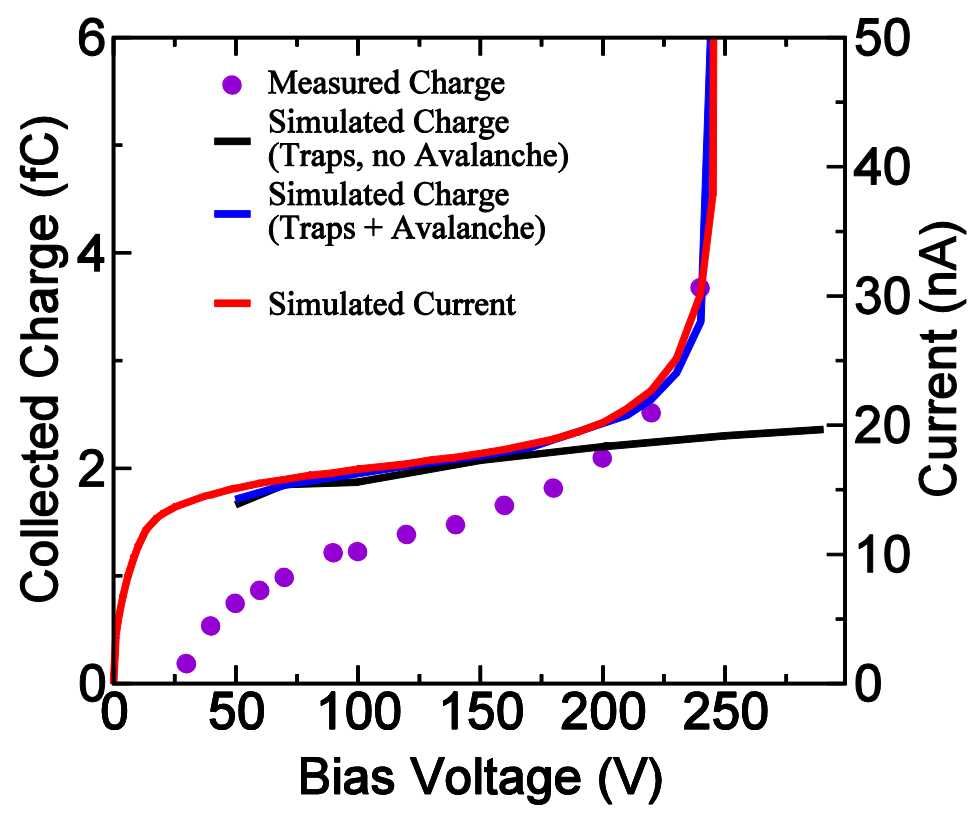

Fig. 4: Simulated vs measured charge released by a m.i.p. for the irradiated $3 D$ sensor described in Section 4. The simulated current signals at the electrodes are post-processed to account for a semi-gaussian filter that emulates a fast, ATLAS-like readout electronics with 20 ns peaking time [12]: signal charge is then extracted from the value of signal peak. The simulated leakage current is also plotted on the right $Y$-axis (red curve).

To understand this effect we performed a first simulation of the irradiated structure in which the effective bulk acceptor concentration and the carrier lifetimes were set accordingly to the values expected after an irradiation fluence of $1 \mathrm{e} 151-\mathrm{MeV}-\mathrm{n}_{\mathrm{eq}} / \mathrm{cm}^{2}$. Even with the impact ionization model switched on, we could not observe any multiplication process since the electric field peaks were not high enough. Then, we performed a more precise simulation in which the 
radiation induced effects on the bulk were accounted for by introducing deep-level traps, according to the "Perugia" model [18].

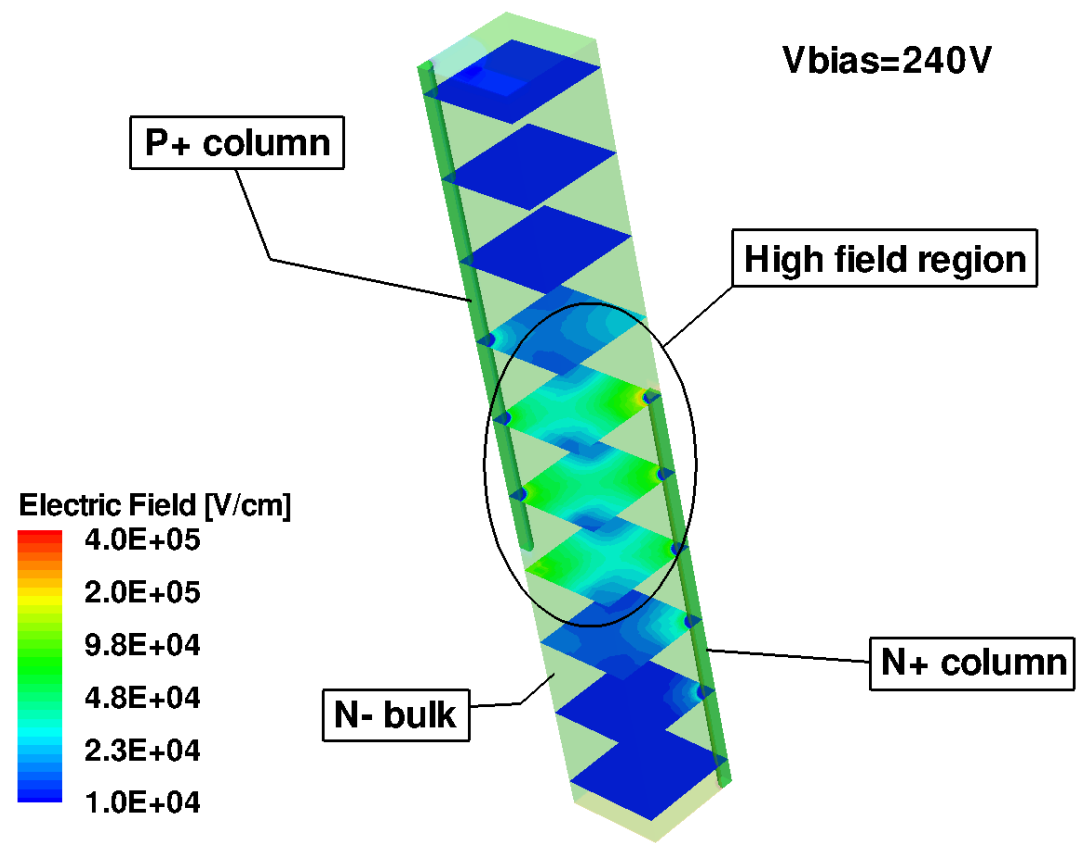

Fig. 5: Electric field maps at several depths into the substrate at a bias voltage of $240 \mathrm{~V}$. The simulation domain is a quarter of the elementary cell considered in Section 4. Irradiation induced traps are inserted into the bulk according to [18].

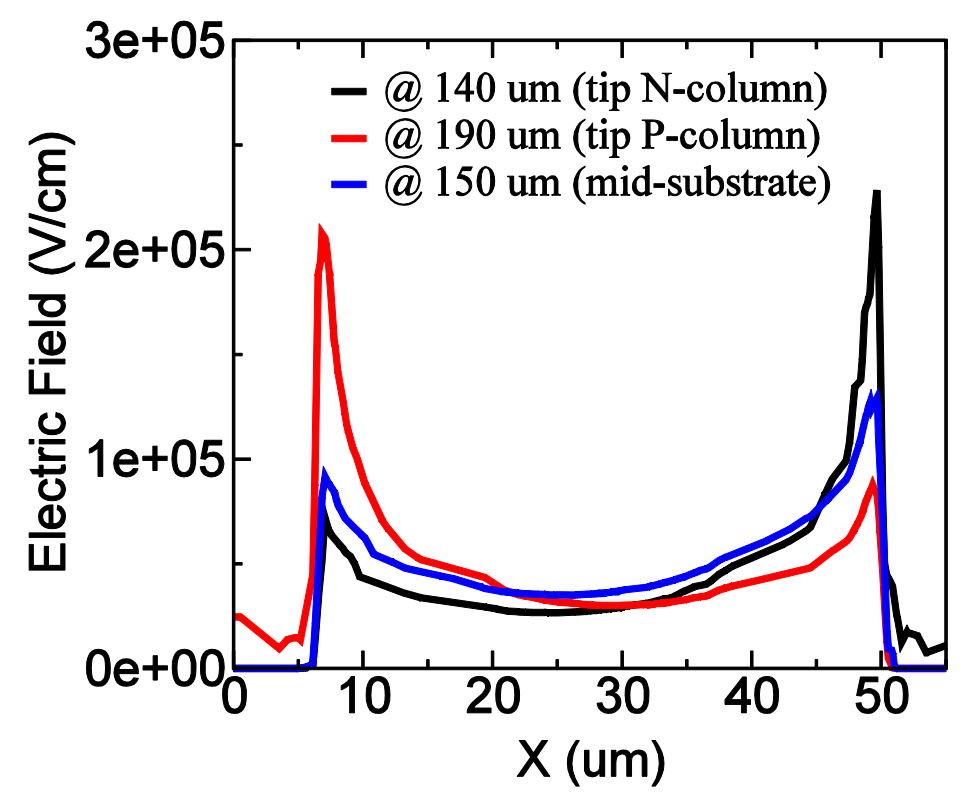

Fig. 6: Electric field profiles derived from Fig. 5, cuts from the p-column (at $X=0 \mu m$ ) to the $n$ column (at $X=40 \sqrt{ } 2 \mu \mathrm{m}$ ) at several depths into the substrate and at a bias voltage of $240 \mathrm{~V}$.

The trap parameters were those of Table II of [18] while the bulk doping concentration and the carrier lifetimes were the same as the in pre-irradiation sensor. Notably, in this 
condition, a multiplication process was visible and a reasonable agreement between experimental data and simulations was found (Fig. 4). In particular, the onset voltage of charge multiplication is accurately predicted, evidence that the electric field distribution is properly modeled. As an example, the electric field maps at a reverse voltage of $240 \mathrm{~V}$ are plotted in Fig. 5 at several depths down into the substrate. It can be seen that the electric fields along both columns in the column overlap region are higher, and pronounced peaks are observed at both column tips. More details can be better appreciated from Fig.6, that shows the electric field profiles along a diagonal starting at the $p+$ column and ending at the $n+$ column at three different depths, corresponding to the column tips and the middle of the substrate. Keeping in mind that the impact ionization coefficient is higher for electrons than for holes, and that in 3D detectors the weighting field is high at both column electrodes (see e.g. [25]), the electric field profiles in Fig. 6 suggest that most of the signal is contributed from the multiplication of electrons at the $n+$ column.

These result, although still preliminary, seem to confirm the possibility to gain insight into the charge multiplication processes taking place in heavily irradiated detectors by using TCAD simulations.

\section{Conclusions}

Since the beginning, simulations of 3D detectors have been deemed as reliable and have been extensively performed in order to get insights of the behavior of the devices. Simulations are performed prior to the production, in order to tune the geometry and technological aspects, and also after the characterization, for a full understanding of the experimental results. With the important exception of the very first papers, such simulations are 3-dimensional, since the physical properties of the devices vary with depth. In this paper we showed in particular how 3D simulations can compute, for the various 3D families, their dead regions, weak points and signal timing, which are properties difficult or even impossible to predict otherwise. If simulations consider suitable models, they are able to reproduce also new effects, such as the observed carrier multiplication in heavily irradiated devices.

\section{References}

[1] S. I. Parker et al., 3D-A proposed new architecture for solid-state radiation detectors, Nucl. Instrum. Methods A, vol. 395, p. 328, 1997.

[2] C.J. Kenney et al., Observation of Beta and X-rays with 3D Architecture Silicon microstrip sensors, IEEE Trans. Nucl. Sci., vol.48, No.2, p.189, 2001.

[3] D. Calvo et al., Thinned epitaxial silicon hybrid pixel sensors for the PANDA experiment, Nucl. Instrum. Methods A, vol. 594, p. 29, 2008.

[4] C. Da Via', et al., 3D active edge silicon sensors with different electrode configurations: Radiation hardness and noise performance, Nucl. Instrum. Methods A, vol. 604, p. 505, 2009.

[5] S.I. Parker, et al., Increased Speed: 3D Silicon Sensors; Fast Current Amplifiers, IEEE Trans. Nucl. Sci., vol.58, No.2, p. 404, 2011. 
[6] M. Garcia-Sciveres et al., The FE-I4 pixel readout integrated circuit, Nucl. Instrum. Methods A, vol. 636, p. S1556, 2011.

[7] M. Barbero, FE-I4 pixel readout chip and IBL module, these proceedings

[8] H. Pernegger, The ATLAS Insertable B-Layer pixel detector, these proceedings

[9] C. Kenney et al., Silicon Detectors with 3-D Electrode Arrays: Fabrication and Initial Test Result, IEEE Trans. Nucl. Sci., vol.46, No.4, p. 1224, 1999.

[10] C. Piemonte et al., Development of $3 D$ detectors featuring columnar electrodes of the same doping type, Nucl. Instrum. Methods A, vol. 541, p. 441, 2005.

[11] C. Piemonte et al., Study of the signal formation in single-type column $3 D$ silicon detectors, Nucl. Instrum. Methods A, vol. 579, p. 633, 2007.

[12] A. Zoboli et al., Double-Sided, Double-Type-Column 3-D Detectors:Design, Fabrication, and Technology Evaluation, IEEE Trans. Nucl. Sci., vol.55, No.5, p. 2775, 2008.

[13] A. Zoboli et al., Functional Characterization of 3D-DDTC Detectors Fabricated at FBK-irst, 2008 IEEE Nuclear Science Symposium Conference Record N34-4

[14] A. Zoboli, Development of radiation detectors with three-dimensional electrodes for future high energy physics experiments, $\mathrm{PhD}$ Thesis, University of Trento, 2009.

[15] A. Zoboli et al., Characterization and modelling of signal dynamics in 3D-DDTC detectors, Nucl. Instrum. Methods A, vol. 617, p. 605, 2010.

[16] D. Pennicard et al., Simulation Results From Double-Sided 3-D Detectors, IEEE Trans. Nucl. Sci., vol.54, No.4, p. 1453, 2007.

[17] D. Pennicard et al., Simulations of radiation-damaged 3D detectors for the Super-LHC, Nucl. Instrum. Methods A, vol. 592, p. 16, 2008.

[18] M. Petasecca et al., Numerical Simulation of Radiation Damage Effects in p-Type and n-Type FZ Silicon Detectors, IEEE Trans. Nucl. Sci., vol.53, No.5, p. 2971, 2006.

[19] I. Perić et al., The FEI3 readout chip for the ATLAS pixel detector Nucl. Instrum. Methods A, vol. 565 , p. $178,2006$.

[20] A. Micelli, et al., 3D-FBK Pixel sensors: recent beam tests results with irradiated devices, Nucl. Instrum. Methods A, vol. 650, p. 150, 2011.

[21] G.-F. Dalla Betta et al., Development of modified 3D detectors at FBK, 2010 IEEE Nuclear Science Symposium, Conference Record N15-3.

[22] C.J. Kenney et al., Active-edge planar radiation sensors, Nucl. Instrum. Methods A, vol. 565, p. 272, 2006.

[23] C. J. Kenney et al., Results From 3-D Silicon Sensors With Wall Electrodes: Near-Cell-Edge Sensitivity Measurements as a Preview of Active-Edge Sensors, IEEE Trans. Nucl. Sci., vol.48, No.6, p. 2405, 2001.

[24] M.Povoli, et al., Slim edges in double-sided silicon 3D detectors, JINST 7 C01015, 2012.

[25] M. Kohler et al., Comparative measurement of highly irradiated $n$-in- $p$ and $p$-in- $n$ 3D silicon strip detectors, Nucl. Instrum. Methods A, vol. 659, p. 272, 2011. 